\title{
Análisis de la influencia del fenómeno stick- slip en los esfuerzos de los cortadores de una broca PDC, mediante simulación por elementos finitos
}

\author{
pags $98-110$ \\ Grupo de Investigación: Diseño Avanzado \\ Línea de investigación: Modelado Computacional

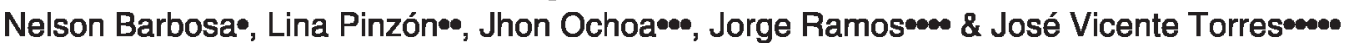

Recibido: 3 de noviembre de 2015

\section{RESUMEN}

Los sistemas rotarios de perforación utilizan brocas PDC 1 para perforar pozos profundos con el fin de explorar y extraer crudo a la superficie. Estos sistemas usualmente sufren fenómenos vibratorios severos que pueden producir fallas en las herramientas de perforación. De manera especifica, las vibraciones torsionales en su condición más crítica, conocida como Stick-Slip, pueden generar delaminación y/o desprendimiento de los cortadores de la broca. El presente artículo, presenta una metodologia para medir los esfuerzos mecánicos presentes como consecuencia de los fenómenos vibratorios de torsión. Adicionalmente, se hace un análisis cualitativo del comportamiento de las herramientas de perforación bajo condiciones de Stick-Slip. Al final, se evidencia la sobrecarga de esfuerzos como consecuencia del fenómeno vibratorio estudiado y se presentan los valores de parámetros aproximados para la operación de la broca sin daños considerables.

Palabras clave: Broca PDC, Vibración torsional, Modelamiento con Elementos Finitos, Stick-Slip.
Aceptado: 6 de noviembre de 2015

\section{ABSTRACT}

Rotary drilling systems use PDC cutters in bits to drill deep wells in order to explore and extract oil to the surface. These systems usually suffers severe vibration phenomena that can lead to failures in drilling tools. Specifically, the torsional vibrations in the most critical condition, known as Stick-Slip, can generate delamination of the bit's cutters. This article presents a methodology for measuring the mechanical stresses present as a result of torsional vibration phenomena. Additionally, a qualitative analysis of the behavior of drilling tools under conditions of stick-slip is presented. Finally. The stress overload as a result of Stick-Slip is evidenced and approximate values for the operating parameters for drilling without significant damage in drilling tools are presented.

Key Words: PDC Drill Bit, Torsional Vibration, Finite Element Modeling, Stick-Slip.

\footnotetext{
- Estudiante coinvestigador del programa de Ingeniería Mecánica. Fundación Universidad de América. nelson.barbosa@estudiantes.uamerica.edu.co

-. IEstudiante coinvestigador del programa de Ingeniería de Petróleos. Fundación Universidad de América. lina.pinzon@estudiantes.uamerica.edu.co

.. Ingeniero Mecánicos, Magíster en Materiales y Procesos. Profesor Investigador Fundación Universidad de América. jhon. ochoa@profesore.uamerica.edu.co

.... Ingeniero de Petróleos, Magíster en Ingeniería. Profesor investigador Fundación Universidad de América. jorge.ramos@ profesores.uamerica.edu.co

....• Ingeniero asesor, Ecopetrol S.A. - ICP

1 Brocas con cortadores de Diamante policristalino compacto (PDC: Polycrystalline Diamond Compact).
} 
LIINEA DE INVESTIGACION: MODELADO COMPUTACIONAL

\section{INTRODUCCION}

Las vibraciones están presentes en todo tipo de sistemas mecánicos y las actividades de perforación no son la excepción. En el proceso de perforación de pozos petroleros, la naturaleza de los fenómenos de vibración depende de las condiciones dinámicas de la broca que son consecuencia de los parámetros de operación seleccionados. Un control inadecuado de los parámetros puede causar fallas prematuras en la broca, desgaste en la sarta de perforación, daños en el equipo de superficie, alterar la estabilidad del agujero y disminuir la tasa de perforación durante la operación (Schen, Snell, \& Stanes, 2005). En la figura 1 se muestra el desgaste de los cortadores de la broca como consecuencia del fenómeno vibratorio de trosión. Las causas físicas que generan este fenómeno están relacionadas a la tensión cíclica, la cual viene acompañada de un movimiento rotacional no uniforme, segundo a la interacción roca-broca y tercero a las fluctuaciones y/o una mala selección de los parámetros de perforación (Fear, Abbassian, Parfitt, \& McClean, 1997):

Figura 1. Mecanismo de desgaste en los cortadores de la broca
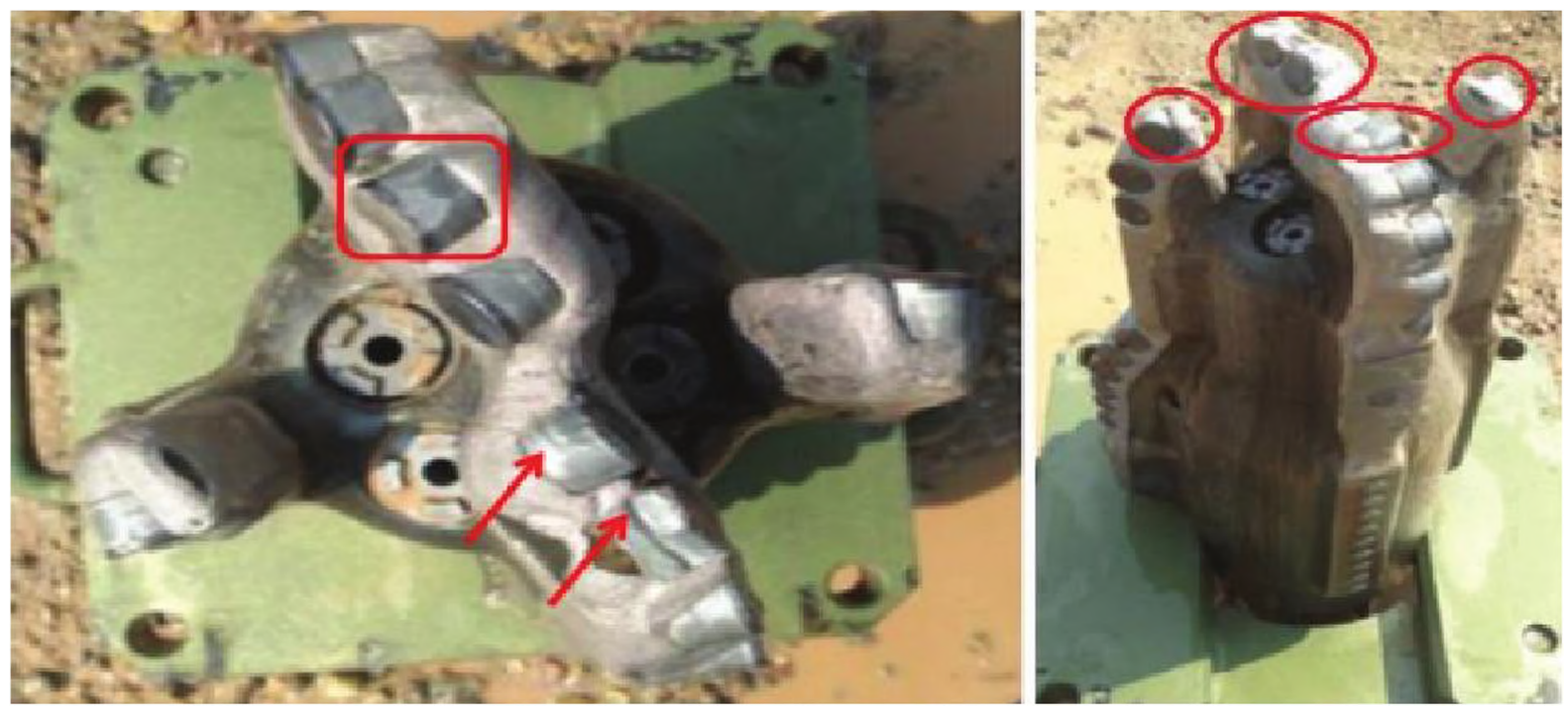

Fuente: (National Oilwell Varco, Análisis dinámico corrido pozo de estudio, sección 12 1/4)

Dependiendo de su dinámica bajo superficie, las vibraciones se pueden clasificar en vibración axial, vibración torsional y vibración lateral. Las vibraciones torsionales son un fenómeno que ocurre en el $50 \%$ de las operaciones de perforación. Aunque las vibraciones laterales han sido identificadas como la causa raíz del daño de una broca PDC, los modelos más actuales de estabilidad en vibración llegan a la conclusión que las vibraciones torsionales por su mayor aparición, se convierten en la principal causa de daño de la estructura de corte de la broca (Pelfrene, Sellami, \& Gerbaund, 2011).
Para el presente documento, las vibraciones torsionales serán consideradas bajo el fenómeno de atascamiento-deslizamiento, conocido como Stick-Slip.

Con el fin de cuantificar el efecto de la vibración torsional sobre los cortadores de una broca PDC se utilizó un software de elementos finitos (ANSYS WORKBENCH) para simular este fenómeno. Se tuvo en cuenta la interacción rocabroca y los parámetros de perforación, como lo son el peso sobre la broca (WOB), el troque aplicado a la broca (TOB) y la velocidad de rota- 
ción de la herramienta (RPM). Estos parámetros son elemento fundamental que dan conocimiento de la magnitud y la propagación de esta vibración. Una vez se lograron los resultados, se evaluaron diferentes escenarios para analizar el comportamiento de los esfuerzos mecánicos en los cortadores de la broca PDC producidos por Stick-Slip.

\section{MATERIALES Y MÉTODOS}

Se estudió una broca PDC de la que se tenían datos censados en servicio. La perforación en la que trabajó la broca corresponde a pozo en donde se presentaron fenómenos vibratorios torsionales conocidos como Stick-Slip. Como criterio de selección de los eventos de stickslip, se utilizó la información suministrada por la compañía National Oilwell Varco (National Oilwell Varco, Análisis dinámico corrido pozo de estudio, sección 12 1/4). De la columna estratigráfica se determinaron las unidades litológicas en donde se presentó el fenómeno y sus propiedades geomecánicas fueron establecidas por medio de la literatura. Se implementaron simulaciones computacionales de interacción de los cortadores de la broca con la formación rocosa y se estudiaron los resultados de esfuerzos y deformaciones obtenidos.

\subsection{Criterio para la selección de las secciones críticas por vibraciones}

La medición de vibraciones torsionales se realizó con una herramienta de almacenamiento en tiempo real (LWD²) llamada BlackBox HD. Este instrumento está ubicado dentro de la broca y tiene la capacidad de medir la variación de la velocidad de rotación en fondo. Con esto valores censados se genera una escala de medida porcentual conocida como el DRPM. Si el valor de DRPM supera el $35 \%$ la situación corresponde a la aparición de Stick-Slip (National Oilwell Varco, "BlackBox Tool, Theory of Operation Downhole"). Por medio de este criterio se extrajeron los parámetros de perforación en fondo responsables de vibraciones torsionales severas y moderadas. Según las mediciones de vibraciones hechas por la compañía NOV ${ }^{3}$ para el pozo, se logró identificar que en la columna estratigráfica el fenómeno Stick-Slip se presentó mayoritariamente al perforar la Formación Carbonera (National Oilwell Varco, Análisis dinámico corrido pozo de estudio, sección 12 1/4) y ésta fue seleccionada como formación de estudio. En la Tabla 1 se observan los datos extraídos de la herramienta clasificados dependiendo de la unidad litológica en donde ocurrió el fenómeno.

\subsection{Propiedades geomecánicas de la formación estudiada}

Para efectos del estudio acá presentado, se consideró que la formación Carbonera se tenía un comportamiento lineal e isotrópico. En la Tabla 2 se presentan las propiedades geomecánicas de las unidades de la formación carbonera, en este caso: la Unidad Arenisca Superior, la unidad Lutita E y la Unidad C1.

\subsection{Geometría de la broca PDC}

La broca empleada para el estudio tenía un diámetro de $81 / 2$ pulgadas; tenía 30 cortadores distribuidos en 6 aletas, 3 principales y 3 secundarias, con cortadores de 13 y 19 mm de diámetro. En la Tabla 3 se presentan las propiedades mecánicas de los materiales de los cuales está construida la broca.

\subsection{Implementación computacional}

Con los parámetros mecánicos, geomecánicos y los estados de carga iniciales necesarios para el modelo, se implementó un modelo computacional basado en un software de elementos finitos. Se cuantificó el impacto de las vibraciones torsionales sobre los cortadores de la broca PDC. Para esto, el modelo se descompuso en tres componentes:

$3 \quad$ National Oilwell Varco 
LÍNEA DE INVESTIGACIÓN: MODELADO COMPUTACIONAL

Tabla 1. Parámetros de perforación que indican la aparición de la vibración torsional

\begin{tabular}{|c|c|c|c|c|}
\hline \multicolumn{5}{|c|}{ Unidad Arenisca Superior / DRPM 35 \% (Moderado) } \\
\hline MD [Ft] & Torque [Klb-ft] & RPM & WOB [KIb] & BBHD_DRPM[\%] \\
\hline 4194.27 & 8.62 & 190.54 & 3.88 & 28.20 \\
\hline 4195.81 & 9.39 & 191.41 & 2.72 & 31.14 \\
\hline 4197.34 & 9.19 & 190.96 & 3.61 & 35.22 \\
\hline 4202.44 & 8.08 & 190.53 & 3.30 & 35.23 \\
\hline \multicolumn{5}{|c|}{ Unidad Lutita E / 35\% < DRPM< 70 \% (Moderado) } \\
\hline MD [Ft] & Torque [Klb-ft] & RPM & WOB [KIb] & BBHD_DRPM[\%] \\
\hline 4202.44 & 8.08 & 190.54 & 3.30 & 35.22 \\
\hline 4203.65 & 8.66 & 190.51 & 3.41 & 42.00 \\
\hline 4234.45 & 9.06 & 191.23 & 4.39 & 38.16 \\
\hline 4260.71 & 6.30 & 191.30 & 4.70 & 42.52 \\
\hline MD [Ft] & Torque [Klb-ft] & RPM & WOB [KIb] & BBHD_DRPM[\%] \\
\hline 5117.79 & 5.56 & 210.77 & 1.82 & 267 \\
\hline 5116.82 & 6.18 & 210.72 & 2.70 & 353 \\
\hline 5130.78 & 6.28 & 211.14 & 1.07 & 246 \\
\hline 5140.29 & 5.04 & 211.34 & 3.02 & 179 \\
\hline
\end{tabular}

Fuente: NOV, Optimización \& Soluciones Integrales. Datos 1ra Corrida pozo de estudio

Tabla 2. Propledades geomecánicas promedio de las Unidades

\begin{tabular}{|l|l|l|l|}
\hline \multicolumn{1}{|c|}{ Propiedad } & \multicolumn{1}{c|}{ Unidad Arenisca Superior } & $23.4 \%$ & $23.45 \%$ \\
\hline Porosidad & $21.2 \%$ & $144.02 \mathrm{lb} / \mathrm{ft}^{3}$ & $148.39 \mathrm{lb} / \mathrm{ft}^{3}$ \\
\hline Densidad & $130.74 \mathrm{lb} / \mathrm{ft}^{3}$ & $2731060 \mathrm{psi}$ & $2934113.43 \mathrm{psi}$ \\
\hline Módulo de Young & $1764529.11 \mathrm{psi}$ & 0.341 & 0.3327 \\
\hline Relación de Poisson & 0.3418 & $6337.42 \mathrm{psi}$ & $6307.40 \mathrm{psi}$ \\
\hline UCS Promedio & $3508.46 \mathrm{psi}$ &
\end{tabular}

Tomado de: (Grupo Central de Tecnologías ECP)

Tabla 3. Propiedades mecánicas de los materiales de la broca

\begin{tabular}{|c|c|c|}
\hline Propledad & Carburo de tungsteno & PDC \\
\hline Densidad & $\begin{array}{l}936.42 \mathrm{lb} / \mathrm{ft}^{3} \\
\text { (AZO Materials, 2015) }\end{array}$ & $\begin{array}{l}219.12 \mathrm{lb} / \mathrm{ft}^{\mathrm{3}} \\
\text { (AZO Materials, 2015) }\end{array}$ \\
\hline Módulo de Young & $\begin{array}{l}99495.88 \text { Ksi } \\
\text { (AZO Materials, 2015) }\end{array}$ & $\begin{array}{l}129083 \mathrm{Ksi} \\
\text { (Bertagnolli \& Cooley, 2001) }\end{array}$ \\
\hline Esfuerzo de fluencia & $\begin{array}{l}60.91 \text { Ksi } \\
\text { (AZO Materials, 2015) }\end{array}$ & $\begin{array}{l}638.16 \mathrm{Ksi} \\
\text { (AZO Materials, 2015) }\end{array}$ \\
\hline Relación de Poisson & $\begin{array}{l}0.22 \\
\text { (AZO Materials, 2015) }\end{array}$ & \begin{tabular}{|l}
0.22 \\
(Yahiaoui, Gerbaud, Paris, Denape, \& Dourfaye, 2013)
\end{tabular} \\
\hline
\end{tabular}

Fuente: Autores 
Interacción Cortador-Roca. Con el objetivo de cuantificar la respuesta mecánica de los cortadores PDC a través de una simplificación de los fenómenos que idealmente se presentan en el proceso de cizallamiento de la formación, se implementó el modelo propuesto por Gerbaud y otros (Gerbaund, Menand, \& Sellami, 2006). Este modelo representa las fuerzas de corte y fricción con tres tipos de cargas estáticas: Fuerza de corte, Fuerza en el bisel y Fuerza detrás del cortador.

A continuación se presentan las ecuaciones involucradas en esos tres tipos de cargas:

Ecuación 1. Ecuación general de la fuerza total sobre el cortador

$$
F=F^{c}+F^{c h}+F^{b}
$$

En donde $F^{c}$ es la fuerza de corte, $F^{c h}$ es la fuerza en el bisel y $F^{b}$ es la fuerza detrás del cortador.

Ecuación 2. Fuerza en la cara del cortador usando el criterio de Mohr-Coulomb

$$
\begin{aligned}
& F_{c}^{c}=\sigma_{0} *\left(1+k^{*} \tan \left(\phi^{\prime \prime}\right) * \tan \left(\omega_{c}\right)\right) * A \\
& F_{n}^{c}=\sigma_{0} *\left(\tan \left(\theta_{f}\right)+k^{*} \tan \left(w_{c}\right)\right) * A
\end{aligned}
$$

En donde $F_{c}^{c}$ es la fuerza de corte en el cortador, $F_{n}{ }^{c}$ es la fuerza normal en el cortador, $k$ es el coeficiente de fricción entre el cortador y la roca y $\phi "$ es el ángulo de fricción entre la roca triturada y la roca virgen.

\section{Ecuación 3. Esfuerzo hidrostático}

$\sigma_{0}=\frac{C_{0}+P_{m}^{*}\left(\sin (\Psi) * \cos (\Psi)+\cos ^{2}(\Psi) * \tan (\varphi)\right)}{\left(1-\tan \left(\theta_{f}\right) * \tan (\varphi)\right) *\left(\sin (\Psi) * \cos (\Psi)-\tan \left(\theta_{f}+\varphi\right) * \sin ^{2}(\Psi)\right)}$

En donde $C_{0}$ es la cohesión de la roca, $\varphi$ es el ángulo de fricción interna de la roca, $P_{m}=$ Presión del lodo, $\psi$ es el ángulo de falla de corte y $\theta_{f}$ es el ángulo de fricción entre el cortador y la formación.

Ecuación 4. Fuerza en el bisel

$$
\begin{aligned}
& F_{c}^{c h}=\sigma_{0} * \tan \left(\varphi^{\prime \prime}\right) * A_{c h} \\
& F_{c}^{c h}=\sigma_{0} * A_{c h}
\end{aligned}
$$

En donde $F_{c}^{c h}$ es la fuerza de corte en el bisel, $F_{n}^{c h}$ es la fuerza normal en el bisel, $\sigma_{0}$ es el Esfuerzo hidrostático en el cortador, $A_{c i}$ elárea de la superficie del bisel proyecta en un plano horizontal y $\phi "$ es el ángulo de fricción entre la roca triturada y la roca virgen.

Ecuación 5. Fuerza en la parte posterior del cortador

$$
\begin{aligned}
& F_{c}^{b}=\sigma_{0} * f\left(\alpha, d, \omega_{d}\right) \\
& F_{c}^{b}=\sigma_{0}^{*} f\left(\alpha, d, \omega_{d}\right)
\end{aligned}
$$

En donde $d$ es la profundidad de corte, $\alpha$ es el ángulo de represión y $\omega_{d}$ es el ángulo de alivio donde recae el cortador con la superficie en contacto con la formación.

Debido a que cada carga tiene dos componentes, una de corte y una normal para los tres tipos de cargas, se realizó un diagrama de cuerpo libre y se obtuvieron dos fuerzas resultantes que fueron ingresadas a la simulación. En la figura 2 Se presenta la simplificación de la formación a perforar propuesta junto con la ubicación final de estas fuerzas.

Respuesta de perforación de una broca de arrastre (PDC). Los parámetros de perforación considerados para para introducir el na simulación fueron el WOB y el TOB. Para incluir estos parámetros, se empleó el método utilizado por Kamel y Yigit (Kamel \& Yigit, 2014) que describe los parámetros que recaen sobre la broca. Consiste en un modelo generalizado de 
un cortador simple, en el que para cada proceso existe una acción de corte y una acción de fricción. A continuación se presentan las ecuaciones del peso y el torque efectivo sobre la broca.

Figura 2. Interacción cortador-roca

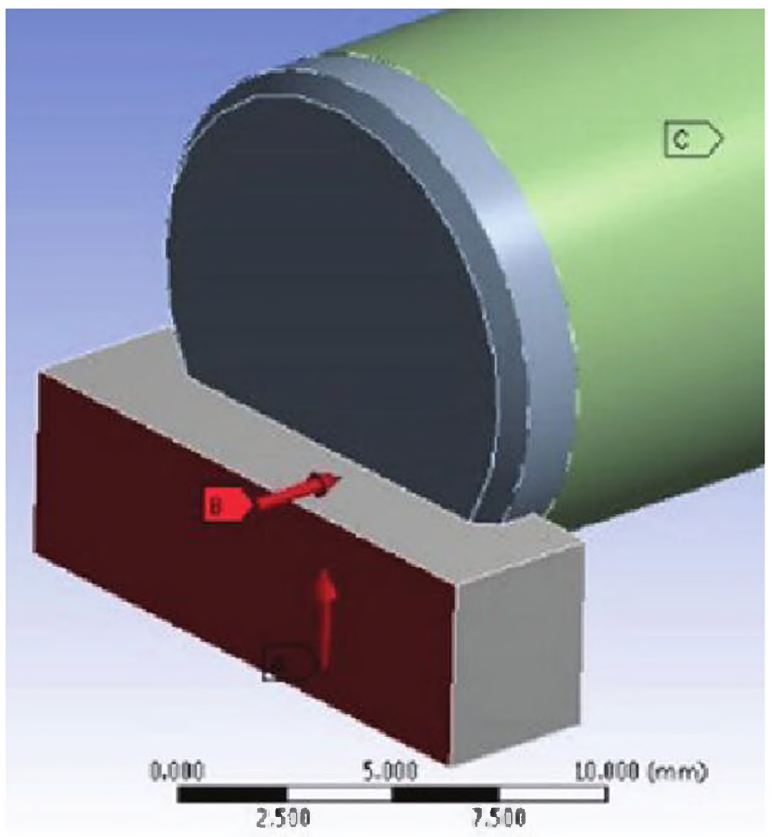

Fuente: Autores

Ecuación 6. Peso efectivo sobre la broca

$$
w=w^{*}+w
$$

En donde $W^{*}$ es el componente del peso asociado con el proceso de corte, $W^{f}$ es el componente del peso asociado con el proceso de fricción. Definidas como:

Ecuación 7. Componente del peso relacionado con la acción de corte

$$
W^{*}=\zeta^{*} \varepsilon^{*} d^{*} a
$$

El valor de $\varepsilon$ corresponde a la resistencia al corte de la roca sin confinar (UCS), $d$ es la profundidad combinada de corte $\left(d=n^{*} d n\right)$, $\mathrm{n}$ es el número de aletas, $d_{n}$ es la profundidad de corte, a es el radio de la broca y $\zeta$ es la razón de la fuerza vertical sobre la horizontal (típicamente, $0.6 \leq \zeta \leq 0.80 .6 \leq \zeta=0.8$ ).

Ecuación 8. Componente del peso relacionado con la acción de fricción

$$
W^{f}=W O B_{\text {sup }}-W^{c}
$$

En donde $W O B_{\text {sup }}$ es el peso sobre la broca en superficie.

Ecuación 9. Torque efectivo sobre la broca

$$
T=T^{c}+T^{F}
$$

En donde $T^{c}$ es el componente del torque asociada con el proceso de corte, $T^{F}$ es el componente del torque asociada con el proceso de fricción. Definidas como:

Ecuación 10. Componente del torque relacionado con la acción de corte

$$
T^{c}=\frac{1}{2} * \varepsilon^{*} d^{*} a^{2}
$$

En donde $\varepsilon$ es el UCS de la roca a perforar, $d$ es la profundidad combinada de corte $\left(d=n^{*} d_{r}\right)$,

$n$ es el Número de aletas, $d_{n}$ es la profundidad de corte y a es el radio de la broca.

Ecuación 11. Componente del torque relacionao con la acción de fricción

$$
T_{f}=T_{\text {sup }}-T_{c}
$$

En donde $T_{\text {sup }}$ es el torque en superficie.

Con este conjunto de ecuaciones, fue posible modelar la acción de perforación de una broca PDC en un estado estable, en donde los fenómenos vibratorios no son tenidos en cuenta.

Incorporación del fenómeno Stick-Slip. Lo efectos de la vibración torsional en su esta- 
do más crítico fueron incorporados al modelo a partir del trabajo presentado por Germay y otros donde se consideran las variaciones que sufre la interacción cortador-roca y la respuesta mecánica de la broca de arrastre cuando el Stick-Slip ocurre (Germay, Denoël, \& Detournay, 2009). Esta perturbación es conocida como los modos de la interacción broca-roca, la cual se clasifica en dos fases: Fase de atascamiento torsional y Fase de deslizamiento torsional como se pueden observar en las Ecuaciones 12 y 13.

Ecuación 12 y 13. WOB y TOB fase atascamiento torsional

$$
\begin{aligned}
& T=T^{c}+T^{F} \\
& W=W^{c}+W^{f}
\end{aligned}
$$

Cuando la velocidad axial de la broca es menor a cero $V<0$, la broca se mueve hacia arriba pero sigue cortando roca. Para esta investigación se asumió que no se presentaban fuerzas del bisel ni fuerzas detrás del cortador debido a que el cortador está desplazado de la superficie del fondo del pozo pero se mantiene perforando. De igual manera el torque y el peso sobre la broca solo tienen el componente relacionado con el proceso de corte ignorando los de fricción cuando esta desliza:

Ecuación 13. Conjunto de ecuaciones fase de deslizamiento torsional

$$
\begin{aligned}
& W=W^{c} \\
& T=T^{c} \\
& F=F^{c} \\
& F_{c}^{c}=\sigma_{0}^{*}\left(1+k^{*} \tan \left(\phi^{\prime \prime}\right) * \tan \left(\omega_{c}\right)\right)^{*} A \\
& F_{n}^{c}=\sigma_{0}^{*}\left(\tan \left(\theta_{f}\right)+k^{*} \tan \left(w_{c}\right)\right)^{*} A
\end{aligned}
$$

Con los parámetros del modelo, condiciones de frontera y los resultados obtenidos de los diferentes modelos de interacción cortador-roca y formación-broca, se definió el estado de cargas sobre la broca PDC. A continuación en la Figura 3. Se muestra el modelo por elementos finitos que incorpora la vibración torsional de acuerdo a la metodología anteriormente planteada.

Figura 3. Modelo por elementos finitos

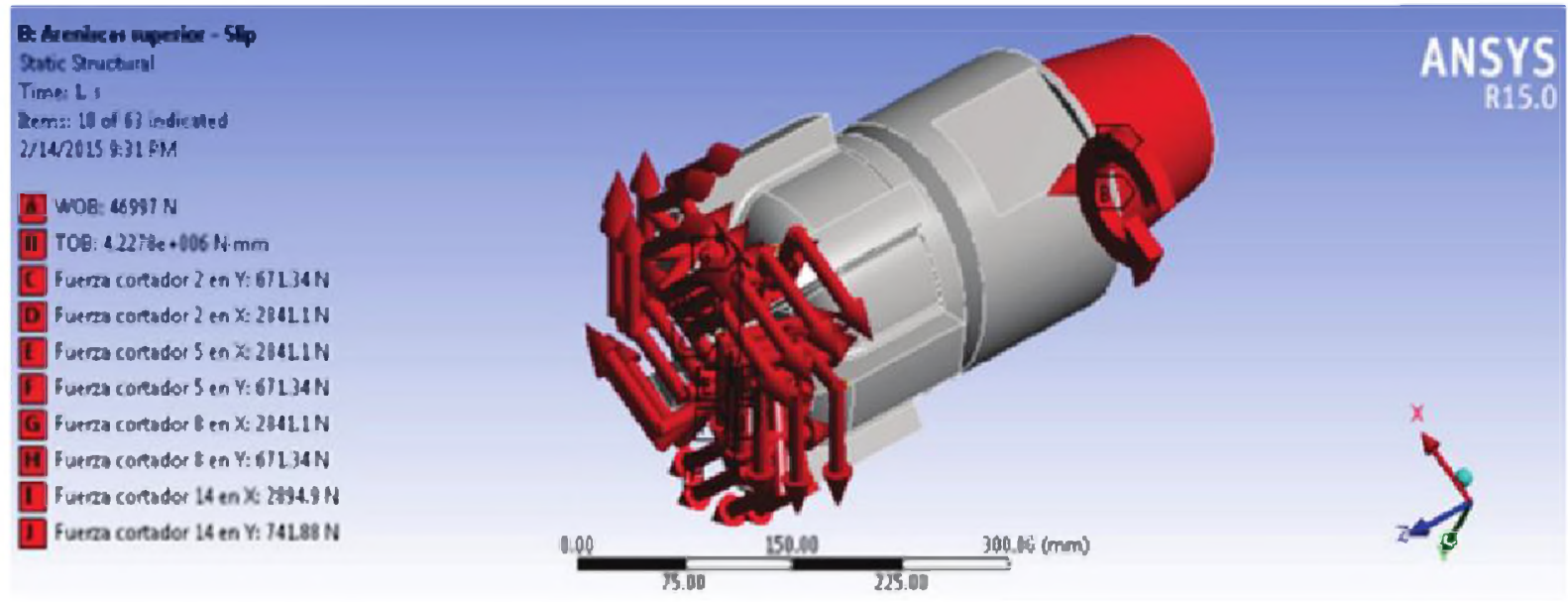

Fuente: Autores 


\section{RESULTADOS}

Con el modelo por elementos finitos definido, se analizaron las diferentes condiciones de operación que se presentaron durante el procedimiento. Fue posible cuantificar de manera aproximada los parámetros de fondo que están actuando sobre la broca. Adicionalmente, se identificó un conjunto de parámetros de perforación para cada Unidad litológica perforada, en donde el fenómeno de vibración torsional pudo ser que generar daños sobre los cortadores.

Para identificar el rango de esfuerzos que pudieran ocasionar daño los cortadores de la broca, se comparó el valor obtenido mediante elementos finitos con el esfuerzo de fluencia del material mediante el concepto de carga máxima permisible (Mott, 2006). A continuación se presenta su ecuación.

Ecuación 14. Carga máxima permisible

$$
C . M . P=\frac{S_{y}}{F . S}
$$

En donde $S_{y}$ es el esfuerzo de fluencia del material y $F . S=$ Factor de seguridad en servicio.
Se cuantificó de manera porcentual la sobrecarga que sufren los cortadores bajo diferentes escenarios estáticos. Además se determinó bajo qué regímenes la herramienta está funcionando o no bajo los esfuerzos admisibles. Para las mediciones de esfuerzos sobre toda la broca, se utilizó un F.S = 3 y para las mediciones del desprendimiento y la delaminación se utilizó un F.S $=1.5$

En la Tabla 4 se presentan los valores máximos de esfuerzos y deformaciones obtenidos en las simulaciones realizadas con los parámetros de perforación que se utilizaron en el pozo; Teniendo en cuenta en qué fase del Stick-Slip se generó y en qué Unidad litológica apareció. Se observa como en la fase de atascamiento torsional generada mientras se perforaba la Unidad Arenisca Superior, el esfuerzo máximo aplicado ronda los $2200 \mathrm{MPa}$, cuando la carga máxima permisible del PDC es de $1466.7 \mathrm{MPa}$, generando una sobrecarga de $48.88 \%$. Debido al exceso de fuerza aplicada, fue necesario optimizar estos parámetros para disminuir el esfuerzo y asi asegurar la vida útil de los cortadores de la broca.

En la figura 4 y a manera de ejemplo, se muestran los resultados obtenidos por mediante Ansys de la distribución de esfuerzos de los cortadores para la sección Lulita E.

Figura 4. Distribución de esfuerzos en los cortadores en la sección Lulita E.

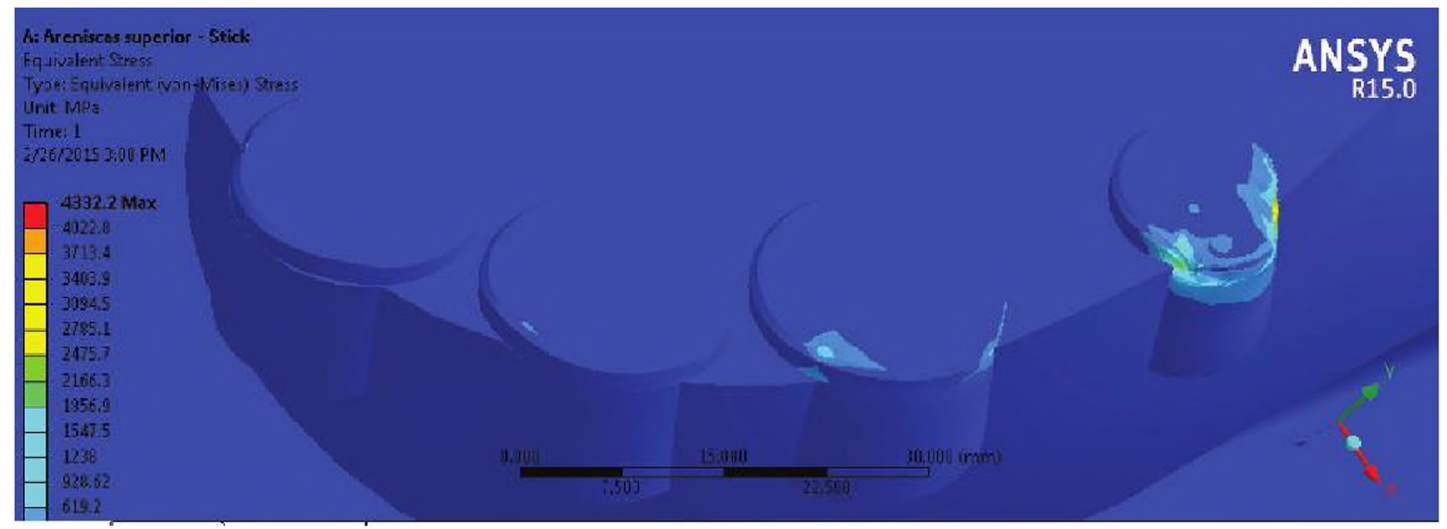

Fuente: Autores 
Tabla 4. Resultados de las simulaciones

\begin{tabular}{|c|c|c|}
\hline \multicolumn{3}{|c|}{ Unidad Arenisca Superior } \\
\hline $\begin{array}{l}\text { Esfuerzo Máximo Fase atasca- } \\
\text { miento [MPa] }\end{array}$ & $\begin{array}{l}\text { Deformación máxima atasca- } \\
\text { miento [mm] }\end{array}$ & \% de carga que sobrepasa la C.M.P \\
\hline 2183.7 & 0.027215 & 48.88 \\
\hline \multicolumn{3}{|c|}{ Unidad Lutita E } \\
\hline $\begin{array}{l}\text { Esfuerzo Máximo Fase Desliza- } \\
\text { miento [MPa] }\end{array}$ & $\begin{array}{l}\text { Deformación máxima desliza- } \\
\text { miento [mm] }\end{array}$ & \% de carga que sobrepasa la C.M.P \\
\hline 1556.2 & 0.097076 & 6.10 \\
\hline \multicolumn{3}{|c|}{ Unidad C1 } \\
\hline $\begin{array}{l}\text { Esfuerzo Máximo Fase Desliza- } \\
\text { miento [MPa] }\end{array}$ & $\begin{array}{l}\text { Deformación máxima desliza- } \\
\text { miento [mm] }\end{array}$ & \% de carga que sobrepasa la C.M.P \\
\hline 870.14 & 0.045182 & -40.68 \\
\hline
\end{tabular}

Fuente: Autores

La optimización propuesta se realizó teniendo en cuenta las curas convencionales utilizadas en campo para mitigar el Stick-Slip (Schlumberguer, 2015). En la Tabla 5 se muestran los parámetros más óptimos que disminuirán el Stick-Slip y así mismo, los esfuerzos aplicados en los cortadores cuando se perforan las Unidades Arenisca Superior y Lutita E. Los parámetros utilizados para perforar la Unidad C1 presentaron un \% de sobrecarga negativo, lo que da como conclusión que el esfuerzo generado no va a dañar las herramientas de corte, es por esto que sólo fue necesario optimizar las Unidades Arenisca Superior y Lutita E.

Tabla 5.Parámetros optimizados

\begin{tabular}{|c|c|c|c|c|}
\hline $\begin{array}{l}\text { Parámetros optimizados } \\
\text { para perforar las 2 } \\
\text { Unidades }\end{array}$ & $\begin{array}{c}\text { Esfuerzo } \\
\text { aplicado [MPa] }\end{array}$ & $\begin{array}{c}\text { Deformación } \\
\text { máxima [mm] }\end{array}$ & $\begin{array}{c}\text { \% de carga que } \\
\text { sobrepasa la } \\
\text { C.M.P }\end{array}$ \\
\hline W[K/b] & T[K/b-ft] & & 0.017615 & -1.33 \\
\hline 14.79 & 6.28 & 1447.1 & $8.39 E-02$ & -11.52 \\
\hline 18.54 & 8.15 & 1297.7 & &
\end{tabular}

Fuente: Autores

Debido a la versatilidad de las curas convencionales propuestas por Schlumberger, se calculó bajo qué parámetros de perforación la broca PDC presentaría daños por sobrecarga. Estos parámetros deberán ser evadidos durante la planeación de la perforación para no incurrir en paradas no planeadas ni en una disminución abrupta de la ROP. Se calcularon utilizando las curas de una manera contraria, es decir, en vez de disminuir el efecto Stick-Slip en fondo, se quiso magnificar su aparición para observar hasta qué punto los cortadores iban a fallar. En la Tabla 6 se muestran los parámetros de perforación que al momento de perforar las diferentes unidades litológicas escogidas, producirán fracturas en los cortadores. 
LÍNEA DE INVESTIGACIÓN: MODELADO COMPUTACIONAL

Tabla 6. Parámetros de perforación más perjudiciales para la broca

\begin{tabular}{|c|c|c|c|c|c|}
\hline \multicolumn{7}{|c|}{ Unidad Arenisca Superior } \\
\hline W[N] & T[Nm] & W[KIb] & T[KIb-ft] & 4332.2 & $\begin{array}{c}\text { \% de carga que } \\
\text { sobrepasa la C.M.P }\end{array}$ \\
\hline 100144.87 & 24038.14 & 22.51 & 17.73 & & 66.14 \\
\hline \multicolumn{7}{|c|}{ Unidad Lutita E } & Esfuerzo aplicado MPa & $\begin{array}{c}\text { \% de carga que so- } \\
\text { brepasa la C.M.P }\end{array}$ \\
\hline W[N] & T[Nm] & W[KIb] & T[KIb-ft] & 4421.8 & 66.83 \\
\hline 101439.58 & 25831.34 & 22.80 & 19.05 & & \% de carga que \\
\hline \multicolumn{7}{|c|}{ Unidad C1 } & & Esfuerzo aplicado MPa & sobrepasa la C.M.P \\
\hline W[N] & T[Nm] & W[KIb] & T[KIb-ft] & 4463.6 & 67.14 \\
\hline 151559.61 & 24292.96 & 34.07 & 17.92 & & \\
\hline
\end{tabular}

Fuente: Autores

Evaluación del desprendimiento y/o delaminación del cortador como consecuencia de la aparición de Stick-Slip. Cuando el fenómeno torsional aparece de una manera severa, se observa que el desgaste es capaz de alcanzar las aletas de la broca y en ciertos casos se presentan fracturas y pérdida de los cortadores (Fear, Abbassian, Parfitt, \& McClean, 1997). Teniendo en cuenta los parámetros utilizados en el pozo, los parámetros optimizados propuestos y los parámetros que generaron daños sobre la herramienta, se midió la capacidad del StickSlip de desprender y/o delaminar los cortadores de la broca PDC empleada.

Para poder identificar si el cortador se delaminó o desprendió, fue necesario investigar las resistencias mecánicas promedio que existe entre las uniones del cortador. Para la unión Vastago-Cuerpo de la broca la resistencia a la fractura promedio es de $338 \mathrm{MPa}$ y la resistencia a la ruptura de la unión Vástago-Cortador PDC promedio es de $1900 \mathrm{MPa}$ (Qian, y otros, 2012).Inicialmente, se midió el esfuerzo máximo aplicado entre las dos uniones y se calculó el porcentaje de carga que sobrepasa a la Carga Máxima Permisible. En la Tabla 7. Se presentan los resultados máximos calculados dependiendo de la Unidad Litológica en donde se produjo Stick-Slip.
Observando los resultados de los porcentajes de carga que sobrepasan a la carga máxima permisible para ambas uniones, se concluye que los cortadores de la broca no presentan delaminación, ni sedesprendimiento cuando se presente Stick-Slip. Existe cierto riesgo en la unión entre el Vástago y el cortador PDC cuando se está perforando la Unidad Arenisca Superior, pero debido a la baja magnitud de este porcentaje no se tendrá en cuenta.

Ya que el efecto Stick-Slip bajó la ROP efectiva en el pozo y fue necesario disminuirlo con la cura convencional, se midió el esfuerzo aplicado en las uniones para observar si dicha optimización los reducía. En la Tabla 8 se muestran los resultados obtenidos. La unidad $\mathrm{C} 1$ no fue optimizada ya que los porcentajes de la carga que sobrepasa la Carga Máxima Permisible están en un rango de operación confiable.

Con esta optimización se observa que los esfuerzos máximos aplicados en cada unión bajaron considerablemente. El porcentaje de carga que sobrepasa la Carga Máxima Permisible fue negativo para los dos tipos de uniones, lo que asegura que si la broca opera bajo los parámetros optimizados que anteriormente fueron planteados, no habrá ningún episodio de delaminación o desprendimiento. 
Tabla 7.Medición de los esfuerzos aplicados con los parámetros del pozo

\begin{tabular}{|c|c|c|c|}
\hline \multicolumn{4}{|c|}{ Unidad Arenisca Superior } \\
\hline Tipo de unión & $\begin{array}{c}\text { Esfuerzo Máximo Fase } \\
\text { atascamiento [MPa] }\end{array}$ & $\begin{array}{l}\text { Deformación maxima } \\
\text { atascamiento [mm] }\end{array}$ & $\begin{array}{c}\text { \% de carga que } \\
\text { sobrepasa la C.M.P }\end{array}$ \\
\hline C-B-Vástago & 195.02 & 0.0088847 & -13.45 \\
\hline Vástago-cortador PDC & 1363.2 & 0.007698 & 7.62 \\
\hline \multicolumn{4}{|c|}{ Unidad Lutita E } \\
\hline Tipo de unión & $\begin{array}{c}\text { Esfuerzo Máximo Fase } \\
\text { Desllzamiento [MPa] }\end{array}$ & $\begin{array}{l}\text { Deformación máxima } \\
\text { desllzamiento [mm] }\end{array}$ & $\begin{array}{c}\% \text { de carga que } \\
\text { sobrepasa la C.M.P }\end{array}$ \\
\hline C-B-Vástago & 139 & 0.072295 & -38.31 \\
\hline Vástago-cortador PDC & 733.48 & 0.073971 & -42.09 \\
\hline \multicolumn{4}{|c|}{ Unidad C1 } \\
\hline Tipo de unión & $\begin{array}{c}\text { Esfuerzo Máximo Fase } \\
\text { Deslizamiento [MPa] }\end{array}$ & $\begin{array}{l}\text { Deformación máxima } \\
\text { deslizamiento [mm] }\end{array}$ & $\begin{array}{c}\text { \% de carga que } \\
\text { sobrepasa la C.M.P }\end{array}$ \\
\hline C-B-Vástago & 95.76 & 0.034118 & -40.68 \\
\hline Vástago-cortador PDC & 403.25 & 0.03914 & -68.16 \\
\hline
\end{tabular}

Fuente: Autores

Tabla 8.Medición de los esfuerzos generados por los parámetros optimizados

\begin{tabular}{|l|c|c|c|}
\hline \multicolumn{5}{|c|}{ Unidad Arenisca Superior } \\
\hline Tipo de unión & $\begin{array}{c}\text { Esfuerzo Máximo Fase } \\
\text { atascamiento [MPa] }\end{array}$ & $\begin{array}{c}\text { Deformación máxima } \\
\text { atascamiento [mm] }\end{array}$ & $\begin{array}{c}\text { \% de carga que } \\
\text { sobrepasa la C.M.P }\end{array}$ \\
\hline C-B-Vástago & 140.32 & 0.006531 & -37.72 \\
\hline Vástago-cortador PDC & 969.68 & 0.005297 & -23.44 \\
\hline \multicolumn{4}{|c|}{ Unidad Lutita E } \\
\hline Tipo de unión & $\begin{array}{c}\text { Esfuerzo Máximo Fase } \\
\text { Deslizamiento [MPa] }\end{array}$ & $\begin{array}{c}\text { Deformación máxima } \\
\text { deslizamiento [mm] }\end{array}$ & $\begin{array}{c}\text { \% de carga que } \\
\text { sobrepasa la C.M.P }\end{array}$ \\
\hline C-B-Vástago & 123.65 & 0.0599 & -45.12 \\
\hline Vástago-cortador PDC & 629.46 & $6.14 E-02$ & -50.30 \\
\hline
\end{tabular}

Fuente: Autores

Como última medida, se cuantificó si los parámetros de perforación que generaban fracturas en los cortadores eran capaces de delaminarlos o desprenderlos. En la Tabla 9 se observan los resultados de los esfuerzos máximos aplicados en las uniones cuando estos parámetros perjudiciales son utilizados. 
LÍNEA DE INVESTIGACIÓN: MODELADO COMPUTACIONAL

Tabla 9.Medición de los esfuerzos generados por los parámetros perjudiciales para los cortadores

\begin{tabular}{|c|c|c|c|}
\hline \multicolumn{4}{|c|}{ Unidad Arenisca Superior } \\
\hline Tipo de unión & $\begin{array}{c}\text { Esfuerzo Máximo Fase } \\
\text { atascamiento [MPa] }\end{array}$ & $\begin{array}{l}\text { Deformación máxima } \\
\text { atascamiento [mm] }\end{array}$ & $\begin{array}{l}\% \text { de carga que } \\
\text { sobrepasa la C.M.P }\end{array}$ \\
\hline C-B-Vástago & 366.02 & 0.18685 & 62.43 \\
\hline Vástago-cortador PDC & 2637.9 & 0.35261 & 108.25 \\
\hline \multicolumn{4}{|c|}{ Unidad Lutita E } \\
\hline Tipo de unión & $\begin{array}{c}\text { Esfuerzo Máximo Fase } \\
\text { Deslizamiento [MPa] }\end{array}$ & $\begin{array}{l}\text { Deformación máxima } \\
\text { deslizamiento [mm] }\end{array}$ & $\begin{array}{c}\% \text { de carga que } \\
\text { sobrepasa la C.M.P }\end{array}$ \\
\hline C-B-Vástago & 360.24 & 0.2113 & 59.86 \\
\hline Vástago-cortador PDC & 1844 & 0.25901 & 45.57 \\
\hline \multicolumn{4}{|c|}{ Unidad C1 } \\
\hline Tipo de unión & $\begin{array}{c}\text { Esfuerzo Máximo Fase } \\
\text { Deslizamiento [MPa] }\end{array}$ & $\begin{array}{c}\text { Deformación máxima } \\
\text { deslizamiento [mm] }\end{array}$ & $\begin{array}{c}\% \text { de carga que } \\
\text { sobrepasa la C.M.P }\end{array}$ \\
\hline C-B-Vástago & 344.84 & 0.18084 & 53.03 \\
\hline Vástago-cortador PDC & 1760 & 0.18976 & 38.94 \\
\hline
\end{tabular}

Fuente: Autores

Como el porcentaje de carga que sobrepasa la Carga Máxima Permisible es positivo y de gran magnitud para cada medición, se concluye que si la broca es corrida bajo los rangos de operación no deseados planteados en las Tabla 6 se presentará delaminación y desprendimiento de los cortadores PDC.

\section{DISCUSIÓN}

La simulación mediante el software de elementos finitos bajo los modelos cualitativos acá propuestos, permite obtener valores aproximados de los esfuerzos estructurales de una broca PDC en operación. Los valores reportados por investigaciones actuales previas, permite validar los resultados arrojados por el software de una manera congruente y así, la optimización propuesta teniendo en cuenta la resistencia a la ruptura de las uniones y del material podrá ser extrapolada a otros estudios sobre vibraciones. Es evidente que el contexto de esta investigación se centró principalmente sobre el fenómeno Stick-Slip, por lo cual se recomienda que el estudio de otros fenómenos vibratorios tales como el salto de la broca y la vibración lateral pueda ser incluido para un análisis completo. La metodología planteada permite tomar acciones correctivas en la perforación de pozos futuros, para de esta manera mitigar los daños prematuros en las herramientas de perforación y las paradas no programadas durante la operación.

\section{REFERENCIAS}

AZO Materials. (03 de 06 de 2015). "Tungsten Carbide - An Overview". Obtenido de http:/l www.azom.com/properties. aspx?ArticleID=1203
Bertagnolli, K., \& Cooley, C. (2001). "Polycrystalline diamond compact (PDC) design methodology utilizing strain energy Capacity". Proceedings of ETCE 2001. Houston.
Fear, M. J., Abbassian, F., Parfitt, S. H., \& McClean, A. (1997). "The destruction of PDC bit by severe Slip-Stick Vibration". SPE/IADC Drilling Conference. Amsterdam. 
Gerbaund, L., Menand, S., \& Sellami, H. (2006). “All comes from the cutter orck interaction". IADC/SPE Drilling Conference. Miami: HAL.

Germay, C., Denoël, V., \& Detournay, E. (2009). “Multiple mode analysis of the self-excited vibrations of rotatory drilling systems". Journal of Sound and Vibrations, 325, 362-381.

Grupo Central de Tecnologías ECP. (s.f.). "Modelo Geomecánico para los pozoz Akacias 10, 17 y 19".

Kamel, J., \& Yigit, A. (2014). "Modeling and analysis of stikcslip and bit bouence in oil well drillstrings equipped with drag bits". Journal of Sound and Vibrations, 333(25), 6885-6899.

Mott, R. L. (2006). “Diseño de elementos de máquinas". México D.F.: Perason Educación.
National Oilwell Varco. (s.f.). "BlackBox Tool, Theory of Operation Downhole".

National Oilwell Varco. (s.f.). Análisis dinámico corrido pozo de estudio, sección 12 1/4.

Pelfrene, G., Sellami, H., \& Gerbaund, L. (2011). "Mitigating Stick-Slip in deep based on optimization of PDC bit desing". SPE/IADC Drilling Conference and Exhibition. Amsterdam.

Qian, J., McMuray, C., Mukhopadhyay, D., Wiggins, J., Vail, M., \& Bertagnolli, K. (2012). "Polycrystalline diamond cutters sintered with magnesium carbonate in cubic anvil press". International Journal of refractory Metals and Hard Materials, 31, 71-75.

Schen, A. E., Snell, A. D., \& Stanes, B. H. (2005). Optimization of Bit Drilling Performance Using a New Small Vibration
Logging Tool'"'. SPDE/IADC Drilling Conference. Amsterdam.

Schlumberguer. (03 de 06 de 2015). “Mediciones precisas $y$ en tiempo real para una perforación productiva". Obtenido de Sensores y optimización de la dinámica de perforación: https://www.slb.com/ /media/ Files/drilling/brochures/mwd/ drilling_dynamics_sensors_ opt_br_spanish.pdf

Yahiaoui, M., Gerbaud, L., Paris, J., Denape, A., \& Dourfaye, A. (2013). "A study on PDC drill bits quality". Wear: An International Journal on the Science and Technology of Friction, Lubrication and Wear, 298-299, 32-41. 